\title{
Identification and Estimation for Autonomous Vehicle Movement Target Based on The Sonar
}

\author{
WANG Ai-bing ${ }^{1, a}$, CHENG Yan ${ }^{2, b}$, LI Han-hao ${ }^{3, c}$, LIU Ya-peng ${ }^{4, d}$, \\ 1,258\# Youyi South ST, Shijiazhuang, Hebei, China, 050091. Hebei Jiaotong Vocational \& \\ Technical College \\ ${ }^{3}$ Rongchang East ST, Beijing, China, 100176 \\ ${ }^{4} 6$ \# Xueyuan Rd, Hongqi ST, Shijiazhuang, Heibei, China, 05000. Hebei Vocational College Of \\ Labour Relations

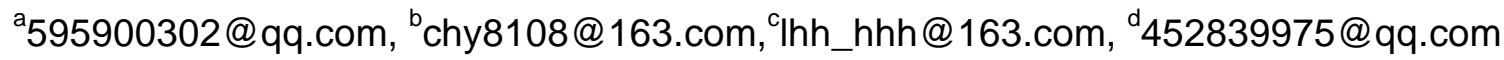

Keywords: Autonomous vehicle; Target identify; Parameter estimation; Simulation

Abstract. Build up the Autonomous Vehicle target uniform motion model, according to the real-time target static character information, give out the reasonable estimation for the uniform motion of the Autonomous Vehicle target by improved least square algorithm, and simulate the parameter estimation, then verify the real-time and the reliability of the algorithm.

\section{Introduction}

Target identification is the key of autonomous vehicle local path planning. The identification includes the static feature of the target shape, size and location. At the same time, the target speed, acceleration and other dynamic characteristics are also captured ${ }^{[1,2]}$. Based on the assumption that the sphere is the autonomous vehicle identification target in the paper, the coordinate of the sphere feature point is captured and identified from the real-time sonar information. In other words, it is how the target static feature identification is completed. According to the target static character time information accumulation, the target is tracked. Then the reasonable estimation for the uniform motion of the Autonomous Vehicle target is generated by improved least square algorithm.

\section{Target Identification}

\section{Sonar Detection Area}

The autonomous vehicle sonar detection radius is $\mathrm{R}$, horizontal open angle is the ${ }^{\beta}$ space stereo area. Within the coordinate system oxyz, the autonomous vehicle target location coordinate is $(x, y, z)$, the autonomous vehicle centroid coordinate is $(0,0,0)$, the sonar is installed in the front of vehicle with the distance to center of mass is $l$, that means the sonar installation location coordinate is $(l, 0,0)$. According to above assumption, the sonar detection area can be calculated as below.

$$
\left\{\begin{array}{l}
\frac{\left|y^{\prime}\right|}{\sqrt{x^{\prime 2}+y^{\prime 2}}} \leq \sin \frac{\alpha}{2} \\
\sqrt{x^{\prime 2}+y^{\prime 2}+z^{\prime 2}} \leq R \\
\frac{\left|z^{\prime}\right|}{\sqrt{x^{\prime 2}+y^{\prime 2}}} \leq \sin \frac{\beta}{2}
\end{array}\right.
$$

Which: $x^{\prime}=x-l, \quad y^{\prime}=y, \quad z^{\prime}=z$ 


\section{Movement Target Identification}

\section{The Sonar Information to the Target Surface Point Conversion}

Each beam can be identified by a three tuple $(i n d e x(i, j)$, length) . It represents the $i$ layer, the $j$ column of the wavelength length of the beam. If the beam three tuple ${ }^{(\text {index }(i, j), \text { length })}$ represent the target surface point location within the coordinate system $o x y z$ as $\left(x_{o b s}^{\prime}, y_{o b s}^{\prime}, z_{o b s}^{\prime}\right)$, then,

$$
\begin{aligned}
& x^{\prime}{ }_{o b s}=l_{c s}+\text { length } \cdot \cos ((i+0.5) \cdot \beta / 3-\beta / 2) \cdot \cos ((j+0.5) \cdot \alpha / 80-\alpha / 2) \\
& y^{\prime}{ }_{o b s}=0+\text { length } \cdot \cos ((i+0.5) \cdot \beta / 3-\beta / 2) \cdot \sin ((j+0.5) \cdot \alpha / 80-\alpha / 2) \\
& z^{\prime}{ }_{o b s}=0+\text { length } \cdot \sin ((i+0.5) \cdot \beta / 3-\beta / 2)
\end{aligned}
$$

Which, ${ }^{l} s$-Sonar installation location to the autonomous vehicle centroid distance, $\mathrm{m}$;

$\alpha$ - Sonar horizontal open angle, rad;

$\beta$ _ Sonar vertical open angle, rad;

\section{Target Feature Points in the Coordinate System}

The target feature points mentioned here is to describe the spatial position of autonomous vehicle target, based on the assumption of the target is a sphere, the sphere center as target feature points.

Assuming that the three sphere points $P_{1}\left(x_{1}, y_{1}, z_{1}\right), P_{2}\left(x_{2}, y_{2}, z_{2}\right), P_{3}\left(x_{3}, y_{3}, z_{3}\right)$ are not in the same large circle, sphere radius is $R$, sphere center is $O\left(x_{0}, y_{0}, z_{0}\right)$. Then the following equations are established

$$
\left\{\begin{array}{l}
\left(x_{1}-x_{0}\right)^{2}+\left(y_{1}-y_{0}\right)^{2}+\left(z_{1}-z_{0}\right)^{2}=R^{2} \\
\left(x_{2}-x_{0}\right)^{2}+\left(y_{2}-y_{0}\right)^{2}+\left(z_{2}-z_{0}\right)^{2}=R^{2} \\
\left(x_{3}-x_{0}\right)^{2}+\left(y_{3}-y_{0}\right)^{2}+\left(z_{3}-z_{0}\right)^{2}=R^{2}
\end{array}\right.
$$

The target feature points $O\left(x_{0}, y_{0}, z_{0}\right)$ can be obtained by using the generalized inverse method of the least square solution of nonlinear equations.

\section{Target Feature Points in the Earth Coordinate System}

Assume that in earth coordinate system $E \xi \eta \zeta$, autonomous vehicle location coordinate is $(x, y, z)$, the attitude angle is $(\varphi, \theta, \psi)$, the autonomous vehicle target feature point coordinates is $\left(x_{o b s}, y_{o b s}, z_{o b s}\right)$. And the target feature points in the coordinate system is $O\left(x_{0}, y_{0}, z_{0}\right)$ 。According to the theory of motion and modeling, the following equations are established,

$$
\left[\begin{array}{c}
x_{o b s} \\
y_{o b s} \\
z_{o b s}
\end{array}\right]=S\left[\begin{array}{l}
x_{0} \\
y_{0} \\
z_{0}
\end{array}\right]+\left[\begin{array}{l}
x \\
y \\
z
\end{array}\right]
$$

Which the transformation matrix $S$ satisfy the following formula

$$
S=\left[\begin{array}{ccc}
\cos \psi \cos \theta & \cos \psi \sin \theta \sin \varphi-\sin \psi \cos \varphi & \cos \psi \sin \theta \cos \varphi+\sin \psi \sin \varphi \\
\sin \psi \cos \theta & \sin \psi \sin \theta \sin \varphi+\cos \psi \cos \varphi & \sin \psi \sin \theta \cos \varphi-\cos \psi \sin \varphi \\
-\sin \theta & \cos \theta \sin \varphi & \cos \theta \cos \varphi
\end{array}\right]
$$

At this point, according to the real-time sonar information, the autonomous vehicle target recognition can be realized within the sonar detection area. 


\section{Parameter Estimation}

Because of the short time period, any movement of the autonomous vehicle target can be considered as a uniform linear motion, so the uniform linear motion model is the most basic model ${ }^{[3]}$. Mathematical Model of Uniform Motion

In the 3D space, the mathematical model of the autonomous vehicle target can be expressed as

$$
\left\{\begin{array}{l}
x=u t+x_{0} \\
y=v t+y_{0} \\
z=w t+z_{0}
\end{array}\right.
$$

Which, $u v w$-Respectively, target/objective velocity (Constant) in the three axis directions

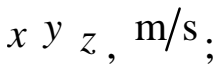

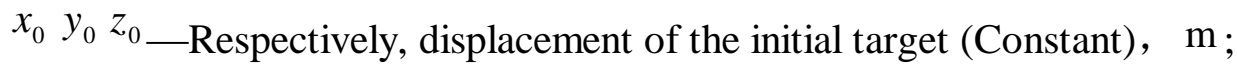

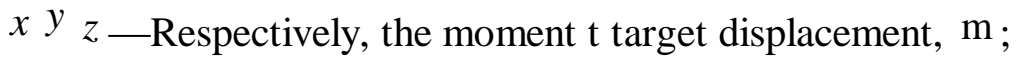

Assuming that the target $x y_{z}$ are independent of the observation sequence of the three axis directions $\left\{x_{i}\right\}\left\{y_{i}\right\}\left\{z_{i}\right\}$, the parameter estimation problem can be considered only in one direction.

\section{Least Squares Estimation}

Through the assumption of sonar information processing, the position coordinates of the autonomous vehicle target are obtained as $\left(x_{o b s}, y_{o b s}, z_{o b s}\right)$, ( short as obs, the same below) And only consider the parameter estimation of the direction of the axis of the coordinate axis $x$, the target motion model can be expressed as $x=u t+x_{0}$. Which $u$ and $x_{0}$ is unknown constant parameter, and a sequence of observations $\left\{x_{i}\right\}_{\text {for }} k$ different $t_{i} \quad(i=1,2 \mathrm{~L} k)$.

A sequence of observations $\left\{x_{i}\right\}_{\text {for }} k$ different $t_{i}$, using a linear regression, then there are $k$ linear equations:

$$
\hat{x}_{i}=u t_{i}+x_{0} \quad(i=1,2 \mathrm{~L} k)
$$

In the form of a matrix: $\hat{X}_{k}=T_{k} A_{k}$

Same as

$$
\left[\begin{array}{c}
\hat{x}_{1} \\
\hat{x}_{2} \\
\mathrm{M} \\
\hat{x}_{k}
\end{array}\right]=\left[\begin{array}{cc}
t_{1} & 1 \\
t_{2} & 1 \\
\mathrm{M} & \mathrm{M} \\
t_{k} & 1
\end{array}\right]\left[\begin{array}{c}
u \\
x_{0}
\end{array}\right]
$$

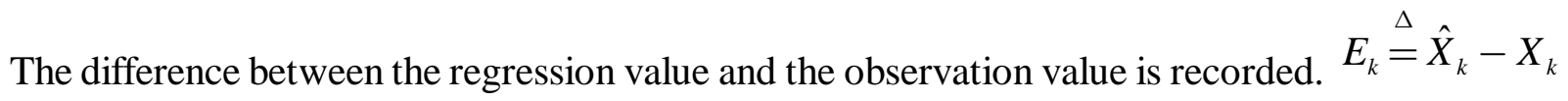

\section{Same as}

$$
\left[\begin{array}{c}
e_{1} \\
e_{2} \\
\mathrm{M} \\
e_{k}
\end{array}\right]=\left[\begin{array}{c}
\hat{x}_{1} \\
\hat{x}_{2} \\
\mathrm{M} \\
\hat{x}_{k}
\end{array}\right]-\left[\begin{array}{c}
x_{1} \\
x_{2} \\
\mathrm{M} \\
x_{k}
\end{array}\right]
$$

Make performance index ${ }^{J_{k}}=\sum_{i=1}^{k} e_{i}^{2}$ Minimum value as $\hat{\mathrm{A}}_{k}$. The optimal value of $A_{k}$ is estimated by the least square method, then the conclusion can be drawn from $\hat{A}_{k}=\left(T_{k}^{T} T_{k}\right)^{-1} T_{k}^{T} X_{k}$.

\section{Improved Least Square Algorithm}

The target state estimation is obtained by using the least square method. If the new measurement data is used to modify the parameter estimates, a recursive formula is used to correct the estimated value. That is the improved least squares algorithm for the estimation of target state estimation. 
Definition $P_{k}=\left(T_{k}^{T} T_{k}\right)^{-1}$, a simple modification of the least squares estimation algorithm based on $\lambda_{\text {is introduced. Take the performance index for }} J_{k}=\sum_{i=1}^{k} \lambda^{k-i} e_{i}^{2} \quad(0<\lambda \leq 1)$, the mean square values from the newer data will be given a greater weight to emphasize the role of the new data, then the solution $\hat{\mathrm{A}}_{k+1}$ at time $k+1$ can be calculated through the solution $\hat{\mathrm{A}}_{k}$ at time $k$ by below formula .

Recursion formula: $\hat{A}_{k+1}=\hat{A}_{k}+G_{k+1}\left[x_{k+1}-\bar{t}_{k+1} \hat{\mathrm{A}}_{k}\right]$

Gain adjustment formula: $G_{k+1}=P_{k} \bar{t}_{k+1}^{T}\left[\lambda I+\bar{t}_{k+1} P_{k} \bar{t}_{k+1}^{T}\right]^{-1}$

Covariance matrix updating formula: $P_{k+1}=\left(P_{k}-G_{k+1} \bar{t}_{k+1}^{T} P_{k}\right) / \lambda$

Which $\bar{t}_{k+1}=\left[\begin{array}{ll}t_{k} & 1\end{array}\right]$, usually take $\lambda=0.85 \sim 0.98$ 。

When the measurement noise is zero, the least square estimation is unbiased. Covariance matrix of measurement noise $R$, then the covariance matrix of estimation error can be drawn by formula $P=\left(T^{T} T\right)^{-1} T^{T} R T\left(T^{T} T\right)^{-1}$.

\section{Simulation Results and Analysis}

In a different time $k$ assumptions have been obtained $k$ observation of target position value, Application of the least square method to the estimation of the dynamic characteristic of target

Assuming that $k$ observation of target position value have been obtained at $k$ different time, the estimation of the dynamic characteristics of the target using the improved least square algorithm。 Simulation results for parameter estimation are shown in Figure 1.
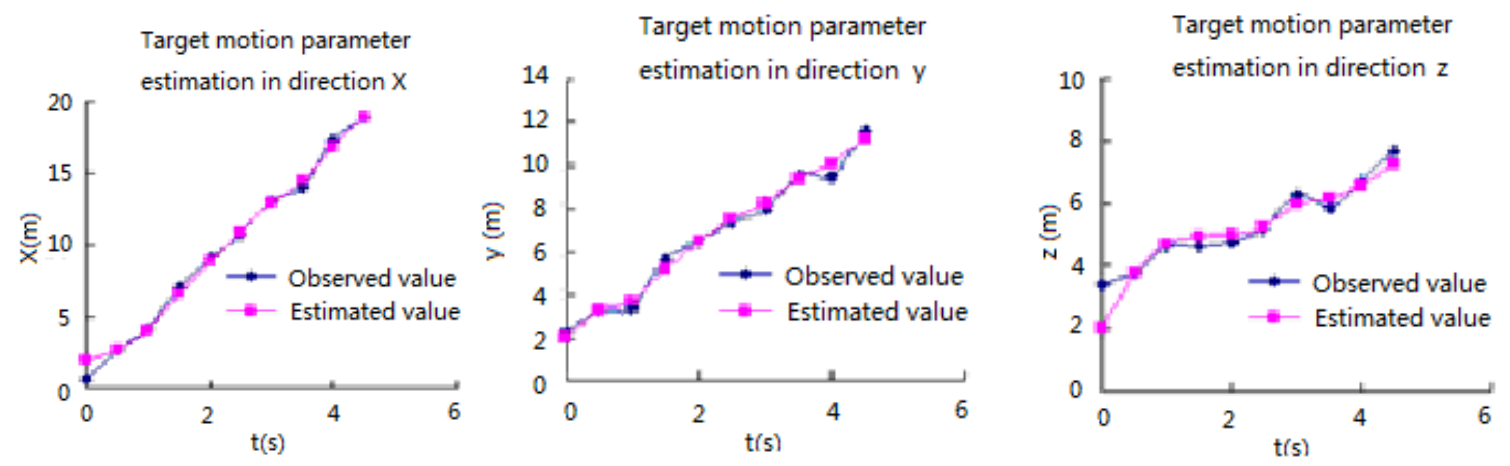

Fig1 Uniform motion parameter estimation

The true value of the state is $u=4.0 、 v=2.0 、 w=1.0 、 x_{0}=1.0 、 y_{0}=2.0 、 z_{0}=3.0$, the observation period is $0.5 \mathrm{~s}$, the observation sequence superimposed the zero mean variance is 0.5 white noise, the initial value of each state is taken as 2.0. After tenth beats, the state estimation value is $\hat{u}=4.0527 、 \hat{v}=2.0472 、 \hat{w}=0.8915 、 \hat{x}_{0}=0.8996 、 \hat{y}_{0}=2.05954 、 \hat{z}_{0}=3.0633$. From Figure 1,we can see that the algorithm can quickly follow the target and effectively suppress the noise.

\section{Conclusion}

In this paper, we give the implementation and simulation results of the improved least square algorithm to improve the algorithm. For uniform linear motion parameter estimation, the algorithm can quickly follow the target, calculate the speed, good real-time performance, high reliability, and can effectively suppress the noise. 


\section{References}

[1] Passino K M. Fuzzy control[M]. Mewlo Park: Addison Wesley Longman Inc, 1998

[2] WANG Jing-qi, CHEN Hui-yan. Survey of land autonomous vehicles [J]. Vehicle and power technology, 2000 (9) : 56-61

[3] WU Yan, QI Fei-hu. A practical algorithm for estimating motion parameters. Academic conference of the Seventh Nation 